deal with the simple and then proceed to the complex, and he would consider only the secondary X-rays. I grant this principle, of course, but I object entirely to the application which he makes of it. It is the $\gamma$ rays which give the simpler effects, and the hardest $\gamma$ rays which give the simplest, for the obvious reason that such rays ignore atomic structure altogether even in the case of the heaviest atoms. The X-rays are soft, and therefore atomic structure influences and complicates the effects to a remarkable degree, as Dr. Barkla's own work shows. A true application of the principle would lead us to work out the laws of the hard $\gamma$ rays first, and then to consider the X-rays in the light of the knowledge we have obtained. This is what $I$ have tried to do. The $\gamma$ rays suggest a corpuscular hypothesis, and on turning to the X-rays it is at once clear that a large proportion of the effects which they show may also be simply explained on the same hypothesis.

Yet I am willing to meet Dr. Barkla even on the narrow ground on which alone he has chosen to risk encounter. He states certain pieces of evidence, numbered $I$ to 9 in his letter, which should show convincingly that a theory of ether pulses is to be preferred to one of neutral pairs. Let us consider these.

No. 2 refers to the equal penetrating powers of primary and secondary rays in certain cases. This is a natural consequence of almost any theory, certainly of a corpuscular one, and the argument may be set aside at once.

No. 3 refers to the equality in the proportion of rays of different penetrating power which are scattered. The theory was given by Prof. Thomson in his "Conduction of Electricity through Gases," and experiments have been made by Dr. Barkla (Phil. Mag., May, 1904) and Mr. Crowther (Phil. Mag., November, 1907). The latter found notable exceptions to the rule, of which no theoretical explanation has yet been offered. The experiments are not easy, and there is enough chance of error to cover a considerable departure from the law, especially considering that no great variation of quality is possible with $\mathrm{X}$-rays alone. In any case, there seems to be no reason for supposing the effect, if a true one, to be a special consequence of the pulse theory. It might well hold for a corpuscular theory, at least over the same limited range.

I have discussed No. 5 in a previous letter. The actual distribution of the secondary scattered rays agrees with the rule deduced by Dr. Barkla in special cases only. In others it does not, and the pulse theory does not say why. One might reasonably expect the rule to be of very partial application, for the secondary kathode radiation has such an extremely asymmetrical distribution that it is hard to believe in a complete symmetry of the remaining secondary radiation. Again, there seems to be nothing irreconcilable with a corpuscular theory.

As regards No. 6, it was asserted by. Dr. Barkla as the result of his experiments that the ratio of the quantity of the secondary radiation to the quantity of the primary depended only on the density of the gas producing it. Prof. Thomson (Phil. Mag., June, Ig06) used the quantitative result as the base of one of three proofs that the number of electrons in an atom was nearly equal to the atomic weight. So far as I can judge, the proofs are not conclusive, and are not generally held to be so. In repeating Dr. Barkla's experiments, Mr. Crowther found the rule to be true only over a very limited range. $\mathrm{C}, \mathrm{N}$, and $\mathrm{O}$ did, indced, give the same ratio, but all other atoms gave different ratios. In the case of $\mathrm{H}$ it was 70 per cent. larger, of $\mathrm{He}$ io per cent., of $\mathrm{S} 40$ per cent., of $\mathrm{Br}$ about 8000 per cent., and so on. Any theory would show a constant effect over so narrow a range. Dr. Barkla considers his result to be evidence of value because it fits in with the result which Prof. Thomson derived from the ratio determined for air ( $\mathrm{N}$ and $\mathrm{O})$; but the strength of the other two proofs of Prof. Thomson's theory is hardly enough to permit this one to be removed and used to support Dr. Barkla's.

Nos. I and 4 refer to the well-known polarisation effects found by Dr. Barkla. The latter has been confirmed by Dr. Haga, who, however, rejects the former. I have already shown that it is not impossible to explain such effects on a neutral pair theory.

Lastly, there are the three statements Nos. 7, 8, and 9 . NO. 2022, VOL. 78]
They refer to certain remarkable effects observed by several workers, particularly M. Sagnac, Dr. Walter, Mr. Adams, and Dr. Barkla himself. Dr. Barkla says that they can be explained on the pulse theory. If any such explanation has been given $I$ have not seen it, and I have not been able to devise any such explanation myself. The secondary X-rays from a substance like copper are homogeneous, and specially able to penetrate screens of the same substance. The homogeneity cannot result from sifting either primary or secondary rays, since it is complete when the radiation leaves the radiator, to say nothing of the difficulty of understanding how outer layers of a substance could sift rays emitted by inner layers of the same substance in the same condition. The effect cannot be due to anything like selective reflection, for then the secondary would be strongly turned back by screens of the same substance. For a similar reason it cannot be a true secondary. It must therefore be a transformed primary, transformed not by the conversion of primary energy into energy of secondary vibrations, but by a true change in its own properties. What can be suggested on the pulse theory as to the nature of this process? Again, in the case of the primary rays, a screen of any one substance has in nearly all cases the power of rendering the rays more penetrating to all other substances, but especially to that substance. It is true that this can be explained by sifting alone, e.g. a substance A might absorb soft rays, a substance $B$ medium rays, and neither hard rays. But it can also be explained by true transformation of the primary as M. Sagnac and Dr. Walter have suggested, yet the transformation must not be accompanied by much scattering of the new radiation. I am aware that $\mathrm{Mr}$. Adams (Amer. Journ. Sci., xxiii., p. 376), unlike M. Sagnac, did not find any effect due to reversing two screens, but $I$ am inclined to think that there is really some transformation of this sort. If that is so the effect will be very hard, if not impossible, to explain on the pulse theory. It is conceivable on a neutral pair theory, since the pair has properties which can be altered without disturbing the velocity and line of flight, so that the primary can be transformed without much scattering. Whether these surmises are correct or not, it seems to me that these particular phenomena give no support to the pulse theory.

Finally, it may be pointed out that the pulse theory will necd radical alteration if it is to explain the asymmetrica effects which Dr. Madsen and I have lately investigated, and it is not clear that the revised theory will fit Dr. Barkla's experimental facts even as well as it does now.

W. H. BRAGG.

The University of Adelaide, South Australia, June 25.

\section{The Discovery of the Weight of the Air.}

THE discovery, in the first half of the seventeenth century, that the air has weight is associated with things of immense importance, for instance, the invention of the barometer and the refutation of the dogma-dear to the false science and the false philosophy of the day-that "Nature abhors a vacuum." In a new edition of the "Essais de Jean Rey," reviewed in Nature of July 9, an attempt is made to assign this discovery to Rey, and, so far, to regard Torricelli, Galileo, Pascal, and Descartes as his disciples. Without claiming to be an authority upon Rey or upon Galileo, I would direct attention to the statement, made in "Galileo-his Life and Work," by J. J. Fahie, that Galileo's way of determining the specific gravity of the air was first described in his letter to Baliani dated March 12, 1613. Rey's "Essais" was published in the year 1630.

Erroneous suppositions regarding Rey being frequently made, I may be allowed to quote Humphry Davy's description of the "Essais" as "a mere logical exercise in physical science." The fact that metals on calcination increase in weight was known to Cardan, Scaliger, Fachsius, Cæsalpinus. Hamerus Poppius, and Libavius, who are mentioned by Rey. For confirmation of the fact he relied upon the statements of his friend "le sieur Brun," and altogether the evidence that Rey made experiments of any value in support of his doctrines is slight.

A. N. Meldrum.

Tannachie, Whitburn, Linlithgowshire, July ir. 